\title{
Enzyme Immunoassay-based Platform For Accurate Detection of Serum Pathological Alpha Synuclein In Parkinson's Disease Patients
}

\author{
Benjamin W. Schlichtmann ${ }^{1,5}$, Naveen Kondru², Monica M. Hepker ${ }^{2}$, Anumantha G. Kanthasamy ${ }^{2,5}$, \\ Vellareddy Anantharam ${ }^{3,5}$, Manohar John ${ }^{4,5,}$, Bhupal Ban ${ }^{6}$, Surya K. Mallapragada ${ }^{1,5,}$, Balaji \\ Narasimhan $1,5,{ }^{*}$ \\ ${ }^{1}$ Department of Chemical and Biological Engineering, Iowa State University, Ames, IA 50011 \\ ${ }^{2}$ Department of Biomedical Sciences, Iowa State University, Ames, IA 50011
}

${ }^{3} \mathrm{PK}$ Biosciences Corporation, Ames, IA 50011

${ }^{4}$ PathoVacs, Incorporated, Ames, IA 50011

${ }^{5}$ Nanovaccine Institute, Ames, IA 50011

${ }^{6}$ Indiana Biosciences Research Institute (IBRI), Indianapolis, IN 46202

\section{ASSOCIATED CONTENT}

\section{A.1 Circular dichroism}

Circular dichroism (CD) spectra from 196-206 nm were measured using a CD spectrophotometer (Jasco J-710, Jasco, Oklahoma City, OK) for $\alpha$ Syn incubated with/without ssMAbs under aggregation conditions, according to the " $\alpha$ Syn incubation with and without ssMAbs" protocol in the experimental section. Spectra of samples were measured at $10 \mu \mathrm{g} / \mathrm{mL}$, using a $10 \mathrm{~mm}$ quartz cuvette. Structural transformation from random coil (negative peaks in this range) to $\beta$-sheet (positive peaks) after aggregation was monitored across all groups (Supplementary Figure 1).

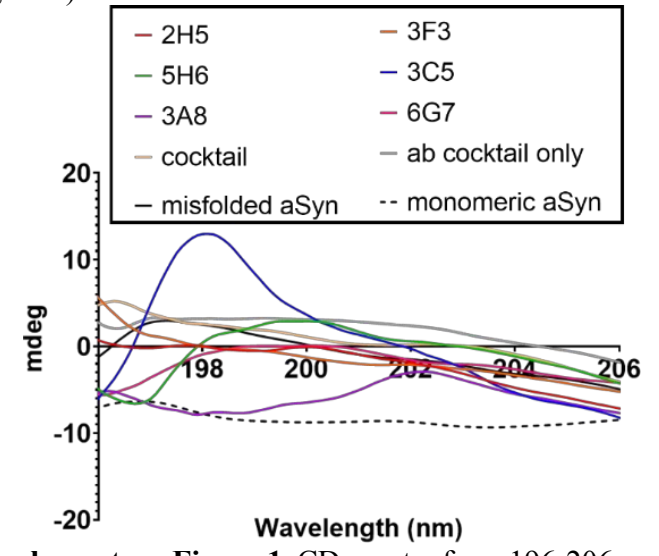

Supplementary Figure 1. CD spectra from 196-206 nm of $\alpha \mathrm{Syn}_{\mathrm{agg}}$ incubated with and without ssMAbs under aggregation conditions from $0-7 \mathrm{~d}$. Samples were measured at $10 \mu \mathrm{g} / \mathrm{mL}$ using a $10 \mathrm{~mm}$ quartz cuvette on a CD spectrophotometer. Whereas $\alpha \mathrm{Syn}_{\mathrm{agg}}$ indicated a positive peak at $198 \mathrm{~nm}$ representative of $\beta$-sheet structure, $\alpha$ Syn incubated with ssMAbs showed a reduction in $\beta$ sheet structure given by negative peaks in the same range, with ssMAb 3A8 showing the most negative peak of all ssMAb groups.

\section{A.2 $\alpha$ Syn RT-QuIC assay}

$\alpha$ Syn RT-QuIC assay was performed based on previously established protocols with small modifications to test activity of antibody on seeding kinetics. ${ }^{32,33}$ In brief, the recombinant $\alpha$ Syn was purified using size exclusion chromatography and anion exchange chromatography and characterized biochemically for purity and activity. The $\alpha$ Syn RT-QuIC was performed using a 96-well clear-bottom plate. The reaction mixture consisted of final concentrations of $40 \mathrm{mM}$ of phosphate buffer ( $\mathrm{pH} 8.0$ ), $170 \mathrm{mM}$ of $\mathrm{NaCl}, 10 \mu \mathrm{M}$ of ThT, and $0.1 \mathrm{mg} / \mathrm{mL}$ of recombinant $\alpha \mathrm{Syn}$. Before each reaction setup, the recombinant $\alpha$ Syn was filtered through a $100-\mathrm{kDa}$ MWCO filter to retain monomer filtrate. The plates were loaded with six 0.8-mm silica beads (OPS Diagnostics, Lebanon, NJ) per well prior to adding reaction components. Each reaction consisted of $5 \mu \mathrm{L}$ of test sample in $100 \mu \mathrm{L}$ of $\alpha$ Syn RT-QuIC reaction mixture per well of a 96-well. The test sample consisted of PD brain or control homogenate diluted 5 logs from $10 \mathrm{wt} \%$ brain homogenate and $0.5 \mu 1$ of ssMAbs at a stock concentration of 500 $\mu \mathrm{g} / \mathrm{mL}$ (final concentration $2.5 \mu \mathrm{g} / \mathrm{mL}$ ) preincubated for approximately $30 \mathrm{~min}$ prior to mixing with reaction mixture. In later experiments with $\mathrm{scFv} 3 \mathrm{~A} 8,2 \mu \mathrm{L}$ of diluted brain homogenates from Dementia with Lewy bodies (DLB) brain homogenates diluted $4 \operatorname{logs}$, and $5 \mu \mathrm{L}$ of scFv $3 \mathrm{~A} 8$ at $500 \mu \mathrm{g} / \mathrm{mL}$ (final concentration $25 \mu \mathrm{g} / \mathrm{mL}$ ) were added, separately, into $93 \mu \mathrm{L}$ of the reaction mixture without pre-incubation. Next, the reactions were performed in a CLARIOstar (BMG LABTECH Inc., Cary, NC) plate reader with alternating 1-min shake and rest cycles (double orbital, $400 \mathrm{rpm}$ ) at $42^{\circ} \mathrm{C}$. ThT fluorescence readings were recorded at excitation and emission wavelengths of 450 and 480 $\mathrm{nm}$, respectively, every $30 \mathrm{~min}$ (Supplementary Figure 2). 

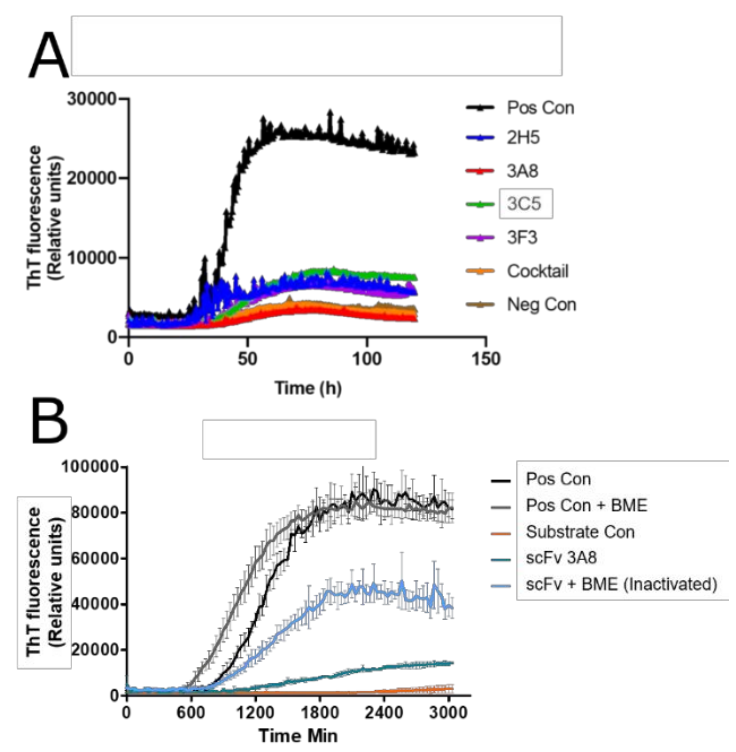

Supplementary Figure 2. A) RT-QuIC assay of top-performing ssMAb IgMs. Pos con: monomeric, recombinant $\alpha$ Syn incubated with PD brain homogenates to seed fibril formation. Neg con: monomeric, recombinant $\alpha$ Syn incubated without $\mathrm{PD}$ brain homogenates, but in the presence of ssMAb 6G7. 2H5, 3A8, 3C5, 3F3, cocktail: IgM ssMAbs pre-incubated with $\mathrm{PD}$ brain homogenates before incubating with monomeric, recombinant $\alpha$ Syn. Cocktail consisted of equal concentrations of all four IgM ssMAbs. B) RT-QuIC assay of scFv 3A8. Pos con + BME: 1mM $\mathrm{BME}$ was added to the positive control to ensure BME did not interfere with the aggregation process. Substrate con: reaction mixture incubated without brain homogenates to ensure aggregation was caused by brain homogenates only. $\mathrm{scFv}+\mathrm{BME}$ (inactivated): $\mathrm{scFv}$ inactivated with $1 \mathrm{mM} \mathrm{BME}$ final concentration prior to adding to wells to ensure reduction in aggregation was associated with scFv $3 \mathrm{~A} 8$-specific activity.

\section{A.3 Dot blot optimization of antibodies}

We performed dot blot assays as described in the materials and methods section to optimize the concentration of the commercial IgG mAbs 5G4 and Syn205. After blocking and rinsing with 1x PBS $3 x$, a range between $10 \mu \mathrm{g} / \mathrm{mL}$ of commercial IgG mAbs $5 \mathrm{G} 4$ or Syn205 in a dilution buffer of 0.1 wt.\% T-20 in 1x PBS was added and the blots were incubated on a tube rotator for $90 \mathrm{~min}$ at RT. After secondary antibody step and adding Opti-4CN substrate, color development was monitored. Analysis of integrated densities for the blots was performed using the gel analysis procedure in ImageJ (Supplementary Figure 3).
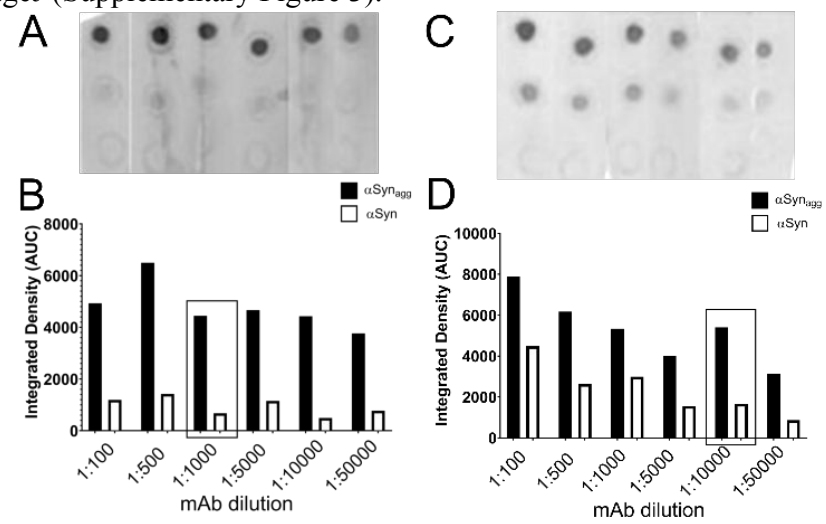

Supplementary Figure 3. Dot blot optimization of Syn205 (A, B) and 5G4 (C, D) commercial mAbs. The optimal dilution, boxed in B), D), indicates the dilution from $1 \mathrm{mg} / \mathrm{mL}$ antibody stock concentration, used for comparison to ssMAbs.

\section{A.4 Sandwich EIA}

A 96 well NUNC ${ }^{\circledR}$ plate was coated with $50 \mu \mathrm{g} / \mathrm{mL}$ purified ssMAb 6G7 in $20 \mathrm{mM}$ sodium carbonate/bicarbonate (pH 9.6) at 4 ${ }^{\circ} \mathrm{C}$ for $18 \mathrm{~h}$. Plates were washed $3 \mathrm{x}$ with wash buffer, and incubated with a blocking buffer of $200 \mu \mathrm{L} 1$ wt. \% BSA in 1x PBS at RT for $7 \mathrm{~h}$, after which blocking buffer was removed. Plates were washed $3 \mathrm{x}$ with wash buffer, and incubated with $150 \mu \mathrm{L} 1 \mu \mathrm{g} / \mathrm{mL} \alpha \operatorname{Syn}_{\mathrm{agg}}$ in a wash/dilution buffer of 0.05 wt. $\%$ T-20 in $1 \mathrm{x}$ PBS at $4{ }^{\circ} \mathrm{C}$ for $18 \mathrm{~h}$. Purified $\alpha \mathrm{Syn}_{\text {agg }}$ was serially diluted 4 -fold from $2,500 \mathrm{ng} / \mathrm{mL}$ to $40 \mathrm{pg} / \mathrm{mL}$ in dilution buffer. Then, $1 \mu \mathrm{g} / \mathrm{mL}$ purified ssMAb was pre-mixed with purified $\alpha \mathrm{Syn}_{\mathrm{agg}}$ in $150 \mu \mathrm{L}$ dilution buffer and subsequently added to the wells. The plates were then incubated at $4{ }^{\circ} \mathrm{C}$ for $18 \mathrm{~h}$. Plates were washed $3 \mathrm{x}$ with wash buffer, then all wells were incubated with $150 \mu \mathrm{L}$ of $10 \mathrm{ng} / \mathrm{mL}$ goat anti-mouse anti-IgM at RT for $6 \mathrm{~h}$. Plates were washed 3x with wash buffer, then $150 \mu \mathrm{L}$ complete ABTS reagent equilibrated to RT was added to each well. The absorbance was read at $\mathrm{OD}_{405} \mathrm{~nm}$ using a SpectraMax ${ }^{\circledR}$ spectrophotometer (San Jose, CA) (Supplementary Figure 4).

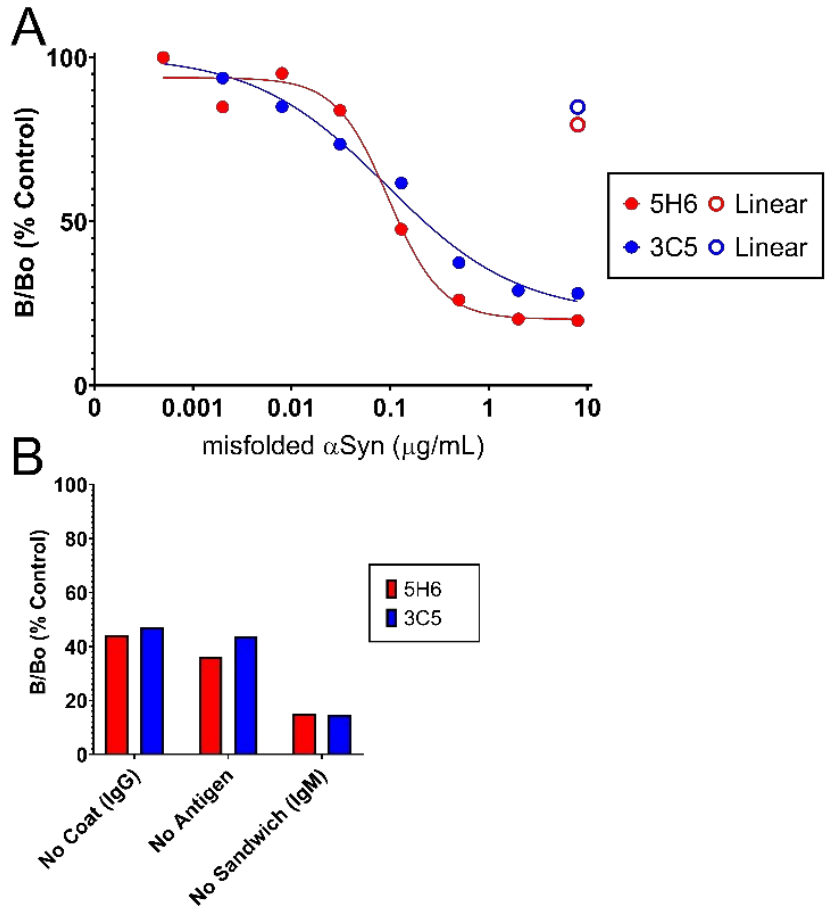

Supplementary Figure 4. Sandwich EIA format of ssMAbs 3C5 and $5 \mathrm{H} 6$ as the sandwich $\mathrm{Ab}$, using ssMAb 6G7 as the capture Ab. (A) Standard curves display similar limits of detection to the indirect format, and similar specificity of $\alpha \operatorname{Syn}_{\mathrm{agg}}$ over $\alpha \mathrm{Syn}$. (B) However, background controls were slightly higher in these assays. For these reasons, this method has not yet been investigated in as much detail as the indirect format reported in the paper. 


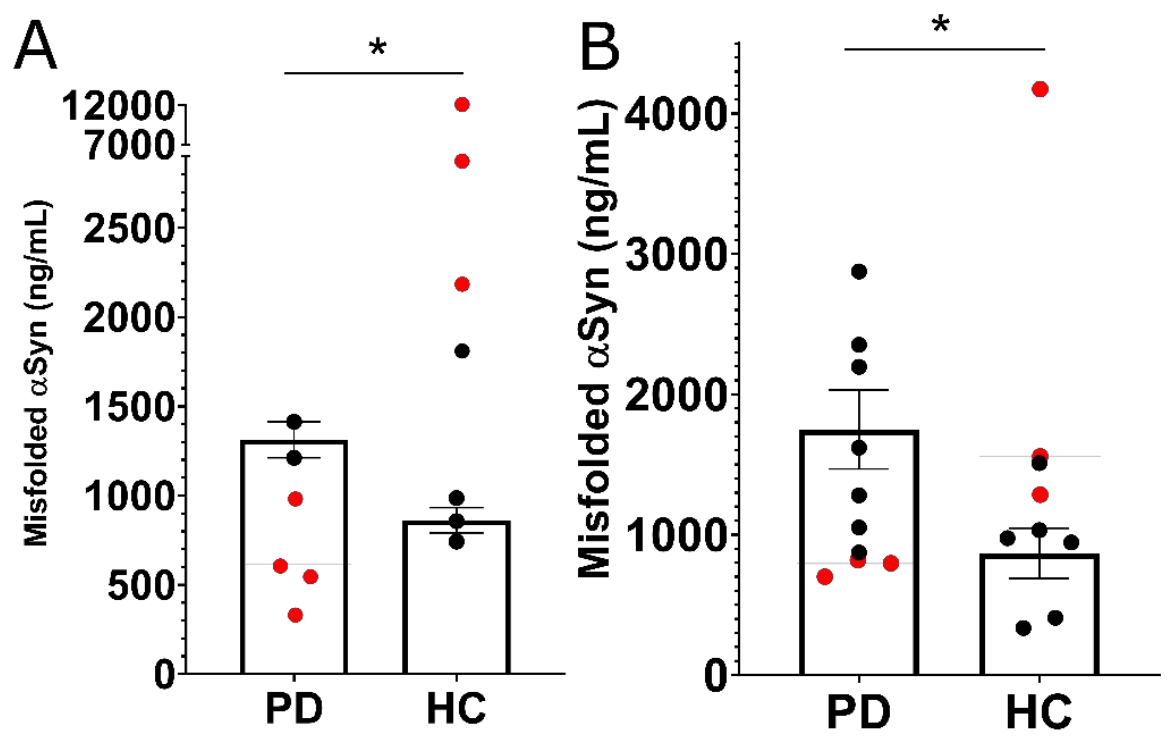

Supplementary Figure 5. Extrapolated concentrations of specimens for A) 50-59 age group and B) 60+ age group. TP PD and TN HC sample $\alpha \mathrm{Syn}_{\mathrm{agg}}$ concentrations were extrapolated individually by assay. Each specimen was averaged across $3 \mathrm{C} 5$ and 5H6 experiments at all dilutions. FN results are shown as red dots in the PD column, and FP results are shown as red dots in the HC column. Outliers from either TP PD or FN HC groups were not included when calculating statistics, but are also shown on the plots. $\mathrm{n}=16$ each for PD and HC samples (10 PD and $8 \mathrm{HC}$ samples 60+, 6 PD and 8 HC samples 50-59). * $p \leq 0.05$ w.r.t. $\mathrm{HC}$

Supplementary Table 1. Patient sample data from BioIVT Incorporated. All specimens came from Caucasian donors. All serum specimens were left-over samples collected for other investigations and were provided to us by the vendor de-identified with only the age, gender, investigations performed, and clinical diagnosis. 


\begin{tabular}{|c|c|c|c|c|c|c|}
\hline $\begin{array}{c}\text { Patient } \\
\text { Sample ID }\end{array}$ & $\begin{array}{c}\text { Hemolyzed } \\
\text { (Approx. Level) }\end{array}$ & $\begin{array}{c}\text { Age } \\
\text { (Years) }\end{array}$ & Gender & Diagnosis & $\begin{array}{l}\text { Hoehn and } \\
\text { Yahr Stage }\end{array}$ & $\begin{array}{c}\text { EIA } \\
\text { Result }\end{array}$ \\
\hline 466524 & Non-Hemolyzed & 51 & Male & Normal Donor & N/A & $\mathrm{FP}$ \\
\hline 299141 & Non-Hemolyzed & 51 & Female & Normal Donor & $\mathrm{N} / \mathrm{A}$ & FP \\
\hline 517612 & Non-Hemolyzed & 52 & Female & Normal Donor & N/A & $\mathrm{TN}$ \\
\hline 517612 & Non-Hemolyzed & 52 & Female & Normal Donor & N/A & $\mathrm{FP}$ \\
\hline 334318 & Non-Hemolyzed & 53 & Female & Normal Donor & N/A & $\mathrm{TN}$ \\
\hline 588179 & Non-Hemolyzed & 54 & Male & Normal Donor & N/A & $\mathrm{TN}$ \\
\hline 595100 & Non-Hemolyzed & 58 & Female & Normal Donor & N/A & $\mathrm{TN}$ \\
\hline 194863 & Non-Hemolyzed & 58 & Male & Normal Donor & N/A & $\mathrm{TN}$ \\
\hline 209551 & Non-Hemolyzed & 62 & Male & Normal Donor & N/A & $\mathrm{FP}$ \\
\hline 516153 & Non-Hemolyzed & 66 & Female & Normal Donor & N/A & $\mathrm{TN}$ \\
\hline 513562 & Non-Hemolyzed & 68 & Female & Normal Donor & N/A & FP \\
\hline 560290 & Non-Hemolyzed & 71 & Male & Normal Donor & N/A & $\mathrm{TN}$ \\
\hline 514892 & Non-Hemolyzed & 75 & Female & Normal Donor & N/A & $\mathrm{FP}$ \\
\hline 411127 & Non-Hemolyzed & 80 & Male & Normal Donor & $\mathrm{N} / \mathrm{A}$ & $\mathrm{TN}$ \\
\hline 514895 & Non-Hemolyzed & 82 & Female & Normal Donor & N/A & $\mathrm{TN}$ \\
\hline 432394 & Non-Hemolyzed & 83 & Male & Normal Donor & $\mathrm{N} / \mathrm{A}$ & $\mathrm{TN}$ \\
\hline 553847 & Non-Hemolyzed & 51 & Female & Parkinson's Disease & 1 & FN \\
\hline 534126 & Non-Hemolyzed & 51 & Male & Parkinson's Disease & N/A & $\mathrm{TP}$ \\
\hline 505782 & Non-Hemolyzed & 52 & Female & Parkinson's Disease & 1 & $\mathrm{FN}$ \\
\hline 519472 & Non-Hemolyzed & 53 & Female & Parkinson's Disease & 1 & FN \\
\hline 518093 & Non-Hemolyzed & 58 & Female & Parkinson's Disease & 2 & FN \\
\hline 559556 & Non-Hemolyzed & 58 & Male & Parkinson's Disease & 1 & $\mathrm{TP}$ \\
\hline 533161 & Non-Hemolyzed & 60 & Female & Parkinson's Disease & 1 & FN \\
\hline 537854 & Non-Hemolyzed & 62 & Male & Parkinson's Disease & 2 & $\mathrm{TP}$ \\
\hline 548067 & Non-Hemolyzed & 66 & Female & Parkinson's Disease & 1 & $\mathrm{TP}$ \\
\hline 562570 & Non-Hemolyzed & 68 & Female & Parkinson's Disease & 1 & $\mathrm{FN}$ \\
\hline 536917 & Non-Hemolyzed & 71 & Male & Parkinson's Disease & 4 & $\mathrm{TP}$ \\
\hline 515748 & Non-Hemolyzed & 75 & Female & Parkinson's Disease & 1 & TP \\
\hline 531500 & Non-Hemolyzed & 77 & Female & Parkinson's Disease & 1 & $\mathrm{TP}$ \\
\hline 547468 & Non-Hemolyzed & 80 & Male & Parkinson's Disease & 1 & $\mathrm{TP}$ \\
\hline 497431 & Non-Hemolyzed & 84 & Female & Parkinson's Disease & 1 & FN \\
\hline 534134 & Non-Hemolyzed & 86 & Male & Parkinson's Disease & 2 & $\mathrm{TP}$ \\
\hline
\end{tabular}

Supplementary Table 2. Standard Curve Percent COV values by B/Bo for ssMAbs 3C5 and 5H6.

\begin{tabular}{|l|c|c|}
\hline B/Bo Standard Curve Percent COV & $3 \mathrm{C} 5$ & $5 \mathrm{H} 6$ \\
\hline
\end{tabular}




\begin{tabular}{|c|c|c|c|c|c|c|}
\hline & & & Intra-assay & Inter-assay & Intra-assay & Inter-assay \\
\hline \multirow{8}{*}{ 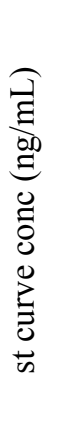 } & No competition & 0 & 0.0 & 0.0 & 0.0 & 0.0 \\
\hline & \multirow{5}{*}{ agg $\alpha$ Syn } & 0.2 & 4.0 & 2.3 & 6.1 & 0.4 \\
\hline & & 2 & 7.4 & 1.1 & 5.9 & 3.7 \\
\hline & & 20 & 8.1 & 4.5 & 6.1 & 5.7 \\
\hline & & 200 & 16 & 12 & 13 & 14 \\
\hline & & 2000 & 31 & 8 & 22 & 16 \\
\hline & \multirow{2}{*}{ monomeric $\alpha$ Syn } & 200 & 5.8 & 2.2 & 8.8 & 4.1 \\
\hline & & 2000 & 5.8 & 2.4 & 12 & 1.8 \\
\hline
\end{tabular}

Supplementary Table 3. Parallelism calculations for ssMAb 5H6 and ssMAb 3C5 across all TP PD samples.

\begin{tabular}{|c|c|c|c|c|c|c|}
\hline \multicolumn{7}{|c|}{ 5H6 Parallelism Calculations } \\
\hline Age (Years) & $1: 3$ & $1: 6$ & $1: 12$ & Avg. & St. Dev & $\mathrm{COV}$ \\
\hline 51 & 1147 & 2623 & 3206 & 2325 & 1061 & 46 \\
\hline 58 & 2200 & 2339 & 1048 & 1863 & 709 & 38 \\
\hline 62 & 2095 & 1131 & 656 & 1294 & 734 & 57 \\
\hline 66 & 1053 & 1068 & 3294 & 1805 & 1289 & 71 \\
\hline 71 & 5364 & 5972 & 2424 & 4587 & 1897 & 41 \\
\hline 75 & 2277 & 2104 & 2129 & 2170 & 93 & 4 \\
\hline 77 & 882 & 2895 & 6912 & 3563 & 3070 & 86 \\
\hline 80 & 723 & 1197 & 1589 & 1170 & 434 & 37 \\
\hline 86 & 1452 & 5299 & 4311 & 3687 & 1998 & 54 \\
\hline TP Sample Avg B/Bo & 27 & 31 & 40 & & & \\
\hline Avg B/Bo of all Samples & 30 & 35 & 43 & & & \\
\hline \multicolumn{6}{|c|}{ Average Sample COV across dilutions } & 48 \\
\hline \multicolumn{7}{|c|}{ 3C5 Parallelism Calculations } \\
\hline Age (Years) & $1: 3$ & $1: 6$ & $1: 12$ & avg & stdev & $\mathrm{COV}$ \\
\hline 51 & 468 & 473 & 567 & 503 & 56 & 11 \\
\hline 58 & 965 & 606 & 116 & 562 & 426 & 76 \\
\hline 62 & 837 & 403 & 118 & 453 & 362 & 80 \\
\hline 66 & 584 & 693 & 995 & 757 & 213 & 28 \\
\hline 71 & 1761 & 1405 & 329 & 1165 & 746 & 64 \\
\hline 75 & 1632 & 869 & 709 & 1070 & 493 & 46 \\
\hline 77 & 331 & 846 & 1314 & 830 & 491 & 59 \\
\hline 80 & 685 & 1243 & 876 & 935 & 283 & 30 \\
\hline 86 & 881 & 1496 & 680 & 1019 & 426 & 42 \\
\hline TP Sample Avg B/Bo & 29 & 39 & 61 & & & \\
\hline Avg B/Bo of all Samples & 36 & 50 & 66 & & & \\
\hline \multicolumn{6}{|c|}{ Average Sample COV across dilutions: } & 48 \\
\hline
\end{tabular}

Supplementary Table 4. Spike and Recovery of four serum specimens tested with ssMAbs 3C5 and 5H6 


\begin{tabular}{|c|c|c|c|c|c|}
\hline \multirow{2}{*}{\multicolumn{2}{|c|}{ Percent Recovery }} & \multicolumn{2}{|c|}{$5 \mathrm{H} 6$} & \multicolumn{2}{|c|}{$3 \mathrm{C} 5$} \\
\hline & & \multirow{2}{*}{$\frac{\text { PD }}{7000}$} & \multirow{2}{*}{$\begin{array}{c}\text { Ctrl } \\
3300\end{array}$} & \multirow{2}{*}{$\frac{\mathrm{PD}}{1300}$} & \multirow{2}{*}{$\frac{\mathrm{Ctrl}}{600}$} \\
\hline \multirow{4}{*}{ 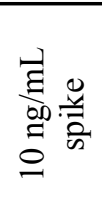 } & $1: 12$ & & & & \\
\hline & $1: 24$ & 560 & -430 & 190 & -63 \\
\hline & $1: 48$ & 490 & 460 & 160 & 110 \\
\hline & $1: 96$ & 430 & 1200 & 88 & 11 \\
\hline \multirow{4}{*}{ 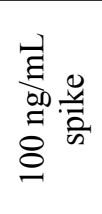 } & $1: 12$ & 970 & 450 & 1400 & 320 \\
\hline & $1: 24$ & 880 & 460 & 300 & 110 \\
\hline & $1: 48$ & 1400 & 700 & 240 & 96 \\
\hline & $1: 96$ & 280 & 390 & 200 & 90 \\
\hline
\end{tabular}


Supplementary Table 5. Complete statistical data of all non-outlier TP PD and TN HC samples for indirect, competitive, semiquantitative tandem EIAs of patient serum specimens incorporating ssMAbs 3C5 and 5H6.

\begin{tabular}{|c|c|c|c|c|c|c|c|c|}
\hline & \multirow{3}{*}{\multicolumn{2}{|c|}{ (All values in ng/mL) }} & \multicolumn{6}{|c|}{ ssMAb } \\
\hline & & & \multicolumn{2}{|c|}{$3 \mathrm{C} 5$} & \multicolumn{2}{|c|}{$5 \mathrm{H} 6$} & \multicolumn{2}{|c|}{ Overall } \\
\hline & & & $\mathrm{PD}$ & Control & $\mathrm{PD}$ & Control & PD & Control \\
\hline \multirow{9}{*}{ 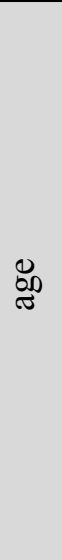 } & \multirow{3}{*}{$50-59$} & mean & 530 & 420 & 2100 & 1300 & 1300 & 860 \\
\hline & & standard error & 30 & 50 & 230 & 190 & 100 & 71 \\
\hline & & range & $500-560$ & $350-510$ & $1900-2300$ & $970-1600$ & $1200-1400$ & $740-990$ \\
\hline & \multirow{3}{*}{$60+$} & mean & 890 & 380 & 2600 & 1400 & 1800 & 870 \\
\hline & & standard error & 90 & 100 & 500 & 280 & 280 & 180 \\
\hline & & range & $450-1200$ & $71-730$ & $1200-4600$ & $600-2500$ & $870-2900$ & $340-1500$ \\
\hline & \multirow{3}{*}{ overall } & mean & 810 & 390 & 2500 & 1300 & 1700 & 870 \\
\hline & & standard error & 87 & 67 & 390 & 190 & 220 & 120 \\
\hline & & range & $450-1200$ & $71-730$ & $1200-4600$ & $600-2500$ & $870-2900$ & $340-1500$ \\
\hline
\end{tabular}

